\title{
Ultra high energy cosmic rays and magnetic fields
}

\section{Todor Stanev*}

Bartol Research Institute, University of Delaware, Newark, DE19716, USA

E-mail: 'stanev@bartol.udel.edui

ABstract: We introduce the puzzles introduced by the existence of cosmic rays of energy above $10^{20} \mathrm{eV}$ and present the effects of the propagation of such particles in extragalactic space. We then outline the additional complications related to propagation in random and regular magnetic fields.

\section{Introduction}

The existence of ultra-high energy cosmic rays (UHECR) has been one of the biggest puzzles in astrophysics during the last forty years. These particles carry macroscopic energies, but little else is known (and is even difficult to imagine) about them: what are they, where and how there are generated, how do they propagate to us? This puzzle became very popular during the last ten years with the prospects of huge new detectors that will be able to collect significant statistics and answer all these questions.

There are two main ideas about the origin of the particles with energies above $10^{20} \mathrm{eV}$. One is the classical astrophysical acceleration scenario (often dubbed bottom-up), such as the one responsible for the majority of cosmic rays. Only extensive and luminous astrophysical objects, such as giant radio galaxies, interacting galaxies or gamma-ray bursts, could generate such particles. The argument goes back to the 1950s when Cocconi [i] suggested that cosmic rays of such high energy have to be of extragalactic origin because their gyro-radii are larger than the dimensions of the Galaxy.

The other class consists of the so called top-down scenarios where UHECR are decay products of supermassive $\left(10^{24}-10^{25} \mathrm{eV}\right) \mathrm{X}$-particles [2i-1]. These X-particles could be emitted from cosmic strings or could be remnants of the early Universe. There are two basic features of the cosmic ray flux generated by these two classes of models. Bottom-up models accelerate charged nuclei, presumably protons, while top-down models generate $\gamma$-rays and neutrinos in the final state of the decay chain. Nucleons are outnumbered by a factor of 30 by these two species. In addition, astrophysical acceleration produces $\mathrm{E}^{-\alpha}$

\footnotetext{
${ }^{*}$ Speaker.
} 
energy spectra with $\alpha>2$, while top-down models generate significantly flatter spectra. There are also some interesting 'hybrid' models [i]i] that combine the two scenarios.

The other element of the puzzle is that nucleons and $\gamma$-rays of such high energy interact and thus lose energy in propagation through the microwave background radiation (MBR). The energy loss should cut off the cosmic ray spectrum. This idea was published almost

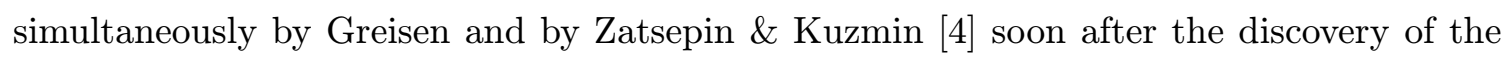
MBR, but three years after the publication of the first detection of a cosmic ray of energy above $10^{20} \mathrm{eV}$ by Linsley 频.

\section{Experimental observations}

The flux of particles of such high energy is small, of the order of one per square kilometer per century, They are observed by the detection of the air showers which their interactions in the atmosphere initiate. There are two different detection methods: air shower arrays and optical detectors. Shower arrays trigger when a large number of spaced particle detectors are hit in coincidence by the shower particles. The energy and type of the primary particle has to be derived from the shower properties. Arrays capable of detecting UHECR have to spread their detectors over many square kilometers. Optical detectors observe the fluorescence of the atmospheric nitrogen excited by the ionization loss of the charged shower particles. The highest energy UHECR observed has more than $2 \times 10^{11}$ charged particles at its maximum. Each charged particle generates about four photons per meter pathlength. Such showers can be observed from large distances.

The highest published contemporary statistics comes from the AGASA array $\left(100 \mathrm{~km}^{2}\right.$ area), although the HiRes optical detector has collected comparable statistics. The main features of the AGASA results are:

- energy spectrum extending above $10^{20} \mathrm{eV}$ with no obvious GZK-like feature [īَi $]$

- large scale isotropy (although the previous lower world statistics showed some tendency of association with the supergalactic plane)

- small scale anisotropy: there are six doublets and a triplet of high energy cosmic rays $\left(\mathrm{E}>4 \times 10^{19} \mathrm{eV}\right)$ that arrive with an angular separation equal to the resolution of the detector $[\bar{i} \bar{i}]]$

The large scale isotropy suggests that the sources of the UHE cosmic rays are isotropically distributed in the Universe and to a large extent contradict to the observed energy spectrum which extends beyond $10^{20}$ without obvious cutoff. Isotropy may also be a result of a strong extragalactic magnetic field in which charged nuclei scatter. Such fields may wash out the source images, especially in the case of low statistics. The small scale clustering, on the other hand, suggests vanishing extragalactic fields.

These exceptional features are challenged by the HiRes preliminary results, which show a cutoff below $10^{20} \mathrm{eV}\left[\begin{array}{l}{[0]} \\ 0\end{array}\right]$ and do not suggest any clustering. On the other hand, the previous version of HiRes, the Fly's Eye, detected a change of the cosmic ray chemical 
composition $[\overline{9}]$, from iron dominated to proton dominated at about $10^{18} \mathrm{eV}$. This observation is not confirmed by the AGASA group. The energy spectra of UHECR detected by the two groups are shown in Fig. 2 after a normalization at $10^{19} \mathrm{eV}$.

Relatively weak limits of the order of $50 \%$ exist on the fraction of $\gamma$-rays above $10^{19} \mathrm{eV}$, but the small world statistics above $10^{20} \mathrm{eV}$ does not allow setting of statistical limits. An extensive review of the experimental techniques and results is this of Nagano \& Watson [i] $\underline{1} 0$

\section{Energy loss in propagation and the formation of the cosmic ray energy spectrum}

There are two energy loss processes for protons in interactions on the microwave background radiation in addition to the adiabatic energy loss due to the expansion of the Universe. At energies exceeding $10^{18} \mathrm{eV}$ protons lose energy in the process $p \gamma \rightarrow e^{+} e^{-}$. This is a resonant process where the cross-section peaks at proton energy about $10^{19} \mathrm{eV}$. At higher energy, when the center of mass energy exceeds $m_{p}+m_{\pi}$ the photoproduction interaction $p \gamma \rightarrow \Delta^{+} \rightarrow p+\pi^{0}\left(n+\pi^{+}\right)$becomes more important. At still higher energy other photoproduction interactions can occur.

Gamma-rays also interact on background photons and generate $e^{+} e^{-}$pairs. In the ultra-high energy range, however, the most important background is not MBR, rather the $\mathrm{MHz}$ radio background, which cannot be observed and has to be theoretically estimated.

Fig. 1 shows the energy loss lengths for protons and $\gamma$-rays as a function of their energy. The $\gamma$-ray energy loss length is based on the radio background model of Protheroe \& Biermann [i] $]_{-1}^{1} \overline{1}$.

The further fate of ultra-high energy $\gamma$-rays depends of the strength of the magnetic field along their path. In fields of order $10^{-9}$ gauss ( $\mathrm{nG}$ ) the electron-positron pair would suffer very strong synchrotron energy loss, that would quickly bring the photon energy in the $100 \mathrm{MeV}$ range. Limits on the mass of the $\mathrm{X}$ particle have been set $[1 \overline{2} 2]$ on the basis of exceeding the observed isotropic $100 \mathrm{MeV} \gamma$-ray background. In fields of $0.01 \mathrm{nG}$ or less the members of the

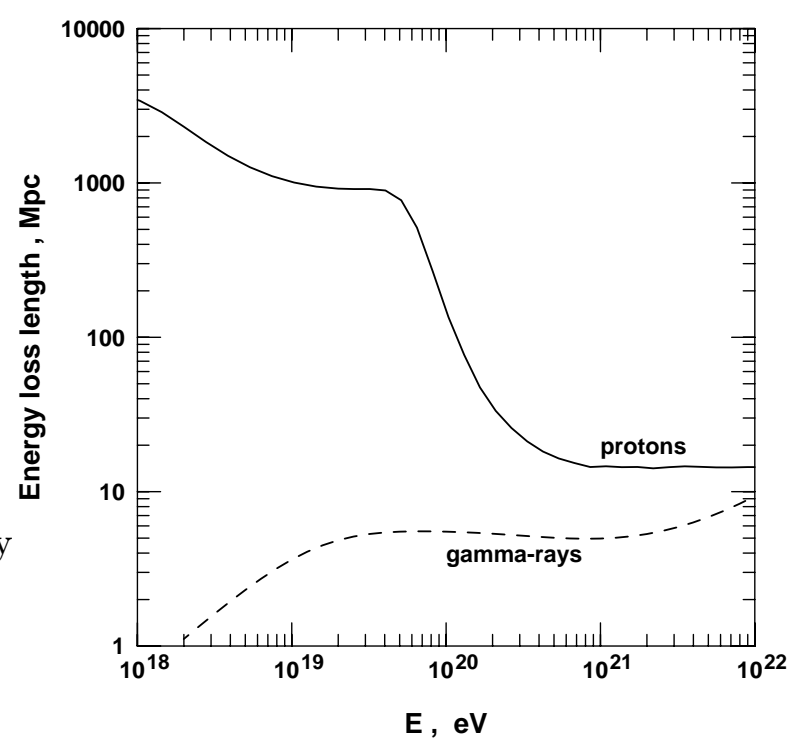

Figure 1: Energy loss length of ultra-high energy protons, solid line, and $\gamma$-rays, dashed line. pair would have inverse Compton scattering, that would lead to much slower electromagnetic cascading, and would not generate too many $100 \mathrm{MeV} \gamma$-rays. In the rest of this presentation we shall discuss the propagation of protons. 
Since the properties of the photoproduction and pair production interactions, as well as the density of the microwave background, are very well known, one can easily calculate the resulting proton energy spectrum for isotropically distributed sources in the assumption of cosmic ray injection (acceleration) spectrum by integration of the energy loss as a function of redshift. An additional assumption, the cosmological evolution of the source luminosity, leads only to second order effects, because ultra-high energy protons emitted at high redshift do not contribute much to the observed UHECR.

Figure 2 shows the result of such integrations for different power law injection spectra with indices $2.00,2.25,0.50,2.75$ and 3.00 in the absence of magnetic fields. The cosmological evolution of the source luminosity is assumed to be proportional to $(1+z)^{3}$. Overlayed are the UHECR spectra reported by the AGASA and HiRes groups. All calculated and the experimental spectra are normalized to a common value at $10^{19} \mathrm{eV}$. The fluxes are multiplied by $\mathrm{E}^{3}$, as it is usually done, to emphasize the shape of the UHECR spectrum.

Apart from the absolute flux normalization of the two experimental groups, the two UHECR do not contradict to each other in shape up to $10^{20} \mathrm{eV}$. One obvious feature is the dip in the vicinity of $10^{19} \mathrm{eV}$ that is first predicted by

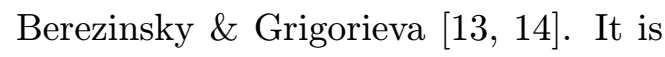
also obvious that it would be very difficult to fit the shape of the observed spectra with injection power law spectra flatter than 2.50. The assumption of steeper spectra would require a mechanism for a decrease of the source contribution at energies below $10^{19} \mathrm{eV}$.

One of the possible mechanism is the effect of extragalactic magnetic fields on the proton propagation, which at these relatively low energies would restrict the

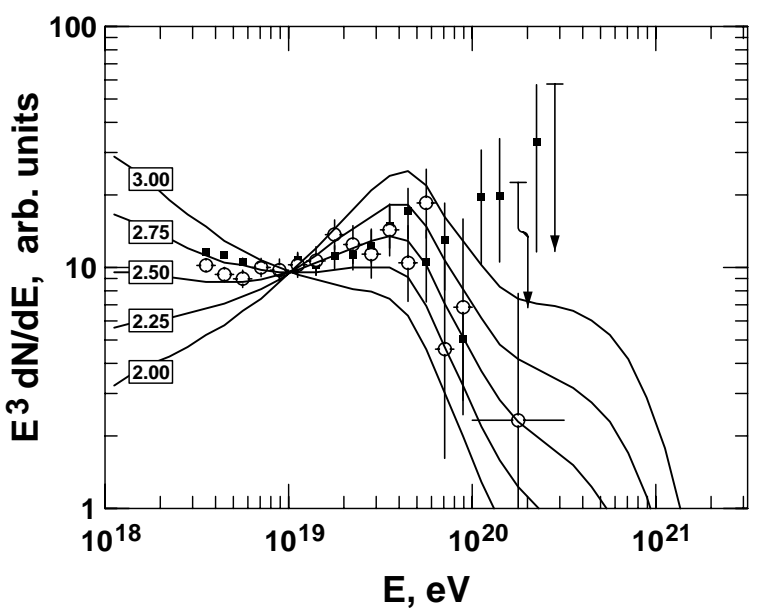

Figure 2: Calculated UHECR spectra from isotropically distributed cosmic ray sources in the assumption of power law injection spectra with indices of 2.00, $2.25,2.50,2.75$, and 3.00 . The spectra measured by AGASA (filled squares) and HiRes (circles) are overlaid. All spectra are normalized to an arbitrary common value at $10^{19} \mathrm{eV}$. distance to the potential UHECR sources
more than the energy loss distance of Fig. 1.

\section{Proton propagation in magnetic fields}

The knowledge of the magnetic fields in extragalactic space is quite limited. The observational technique is the measurement of the Faraday rotation, which also requires knowledge of the electron density between the source and the observer, Very high fields, of order of and exceeding $1 \mu \mathrm{G}$, have been observed in clusters of galaxies [1] ${ }_{1}^{1}$ in on Mpc scales. The average random extragalactic field does not exceed one $\mathrm{nG}$ if the coherence length $l_{c}$ of the field is $1 \mathrm{Mpc}$, of order of the average distance between galaxies. 
Even such relatively low field strengths may prove to be important. For comparison, the gyroradius of $10^{20} \mathrm{eV}$ proton in $1 \mathrm{nG}$ field is $100 \mathrm{Mpc}$. Because of random walk the $10^{20} \mathrm{eV}$ proton would be deflected from the position of the source at angle

$$
\theta(d)=2.5^{\circ}\left(\frac{d}{100 \mathrm{Mpc}}\right)\left(\frac{l_{c}}{\mathrm{Mpc}}\right)^{1 / 2},
$$

where $l_{c}$ is the coherence length of the field.

Even more important is the increased pathlength $\Delta d$, and respectively energy loss, due to the random walk.

$$
\Delta d=47 \mathrm{kpc}\left(\frac{E}{10^{20} \mathrm{eV}}\right)^{-2}\left(\frac{d}{100 \mathrm{Mpc}}\right)^{2}\left(\frac{B}{\mathrm{nG}}\right)^{2}\left(\frac{l_{c}}{\mathrm{Mpc}}\right)
$$

Since the dependence on energy, distance and magnetic field strength is quadratic, the pathlength could easily double the the straight propagation distance to the source. At energies as low as $10^{18} \mathrm{eV} \Delta d$ may exceed the distance to the horizon and prohibit cosmic rays from reaching us.

The increased pathlength is equivalent to time delay - $47 \mathrm{kpc}$ corresponds to an increase of the travel time by $1.5 \times 10^{5} \mathrm{yr}$. This is extremely important if the UHECR production is impulsive, as it would be in gamma-ray bursts. $10^{19}$ protons accelerated in a nearby (100 $\mathrm{Mpc}$ is a redshift of only 0.025$)$ gamma-ray burst would arrive 15 million years later than the $10^{20}$ particles.

Ref. [i] $\left.\underline{1}_{1}^{\overline{6}}\right]$ defines a particle 'horizon' and studies it by propagating protons with energy loss in $1 \mathrm{nG}$ random field. The horizon corresponds to the distance where less than $1 / e$ of the protons arrive with more than one-half of their energy at injection. Figure 3 shows the ratio between the horizon and the energy loss length of Fig. 1 for particles of energies between $10^{19}$ and $10^{20} \mathrm{eV}$. While at energy above $10^{20} \mathrm{eV}$ the proton horizon more or less matches the energy loss distance, at lower energies it is significantly smaller. At energies above $10^{19} \mathrm{eV}$ we do not see the $\mathrm{E}^{-2}$

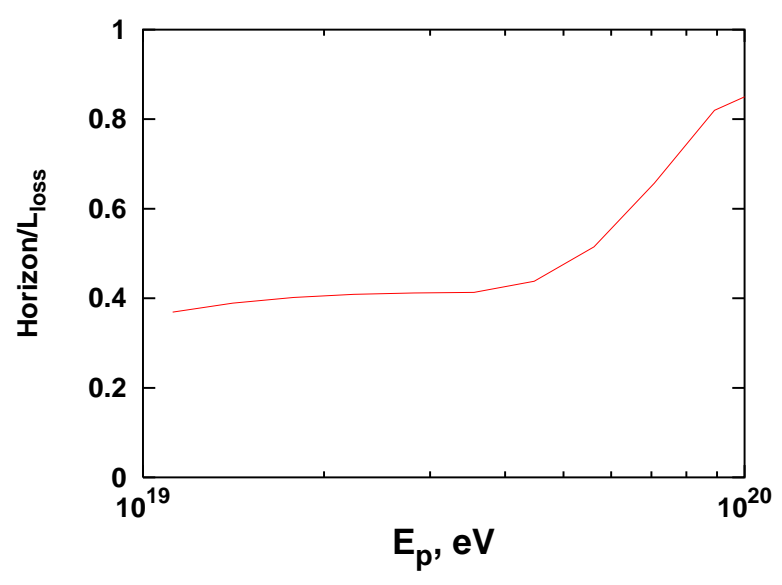

Figure 3: Ratio between the proton horizon and the energy loss length of Fig. 1. See text for the definition of horizon. behavior suggested by the pathlength energy dependence and the horizon is essentially constant at about 370 Mpc. A field strength lower by one order of magnitude $(0.1 \mathrm{nG})$ generates only a slightly higher horizon of $420 \mathrm{Mpc}$ at $10^{19} \mathrm{eV}$. Stronger fields lead to a decrease of the horizon at lower energy.

It would be very interesting to extend such a calculation to lower energy and see when the proton horizon starts exceeding the size of the Universe. Unfortunately this would be very uncertain result, since it depends strongly on the cosmological evolution of the extragalactic magnetic fields, which is totally unknown. 


\section{Proton propagation in ordered magnetic fields}

The main effect of $n G$ strength random magnetic fields on proton propagation is the random walk that delays the particle propagation, increases its pathlength and respectively energy loss. Since the effect is energy dependent, it could lead to additional changes of the injected proton spectrum, which are not accounted for in propagation calculations in non-magnetic environment.

The assumption that coherent magnetic fields extending over $10 \mathrm{Mpc}$ scales exist in the Universe leads to more complications. The main reason is that the proton motion in such fields is different from that in random fields. The random walk is replaced by gyrations around the magnetic field lines, that could speed up or increase the delay in the proton propagation, depending on the pitch angle of the proton. One important consequence is that this motion increases the particle density in the region where the magnetic field is coherent. Since the containment of protons is energy dependent, the spectrum of the protons that reach the same distance from the source depends on the position of the observer relatively to the magnetic structure.

Some simple examples are developed and calculated in Ref. [i] $\left[\begin{array}{l}\overline{1} \\ \overline{1}\end{array}\right]$. In one of them protons are emitted isotropically at the center of a $20 \mathrm{Mpc}$ sphere, which is at the origin of the coordinate system. A magnetic wall of width $3 \mathrm{Mpc}$ is centered on the 'yz' plane. The magnetic field points in ' $\mathrm{z}$ ' direction and its strength inside the wall is $10 \mathrm{nG}$. The field decreases exponentially outside the wall. It is accompanied by a random field with strength one-half that of the regular field.

Figure 4 shows the spectra of particles that reach patches centered on the $\mathrm{z}=20 \mathrm{Mpc}$ (front) and $\mathrm{y}=20 \mathrm{Mpc}$ (top) points on the sphere. The particles that reach the front patch (travel inside the magnetic wall) is enriched with low energy particles. An observer located at this position would derive an $\mathrm{E}^{-3.5}$ injection spectrum and a local source from his observations. An observer in the 'top' position, where protons have to traverse the magnetic fields lines, would see a spectrum almost totally lacking particles below $10^{20} \mathrm{eV}$. He may derive an $\mathrm{E}^{-1}$ injection spectrum. The only energy range where the spectrum is not drastically changed is above $10^{20}$, where observers in either position see only the standard energy loss from propagation

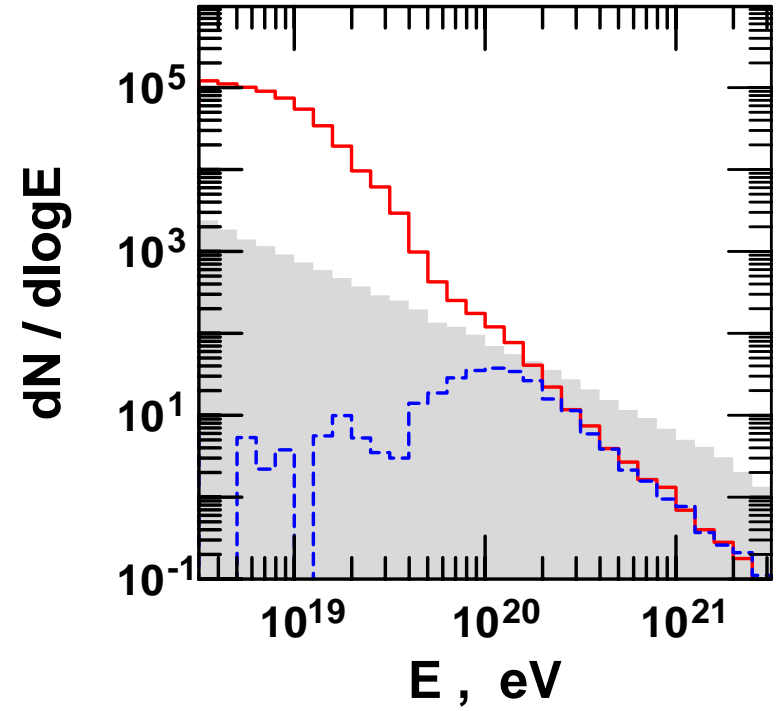

Figure 4: Spectra of protons that reach patches centered on the $\mathrm{z}=20 \mathrm{Mpc}$, solid histogram, and $\mathrm{y}=20$ $\mathrm{Mpc}$, dashed histogram. The shaded histogram shows the injection spectrum. on $20 \mathrm{Mpc}$. All other different locations on the sphere see intermediate spectra, but none of them sees the protons that would reach 
the observers in the absence of the regular field.

Other source positions lead to qualitatively similar, but quantitatively different changes of the observed proton fluxes.

One relatively safe case of cosmic ray scattering in ordered magnetic fields is the galactic field. The measurements of the Faraday rotation of galactic pulsars shows a local strength of $\sim 2 \mu \mathrm{G}$ and field direction toward galactic longitude of $\ell=90^{\circ}$. The field has a reversal at a distance of about $500 \mathrm{kpc}$ in the direction of the galactic center $\left(\ell=0^{\circ}\right)$ and possibly another reversal at about $3 \mathrm{kpc}$ in the opposite direction.

The models of the large scale structure of the galactic field are that it is similar to the distribution of matter, i.e. has a spiral form with a most likely $\pi$ symmetry in the bisymmetric model. No magnetic field component normal to the galactic disk has been detected, but it is possible that it exists at a level not higher than $0.3 \mu \mathrm{G}$. More general estimates of the total magnetic field strength give much higher values of $5-6 \mu \mathrm{G}$. This requires the introduction of strong random field and possibly a halo field that has different structure from the bisymmetric field of the galactic plane.

Figure 5 shows an example of how the motion of $10^{18} \mathrm{eV}$ protons in the Galaxy is influenced by the galactic magnetic field. In this example $10^{18} \mathrm{eV}$ protons are injected isotropically at the $4 \mathrm{kpc}$ circle and their trajectories are followed in the magnetic field model, which includes a random component with strength of one-half of that of the regular field. The figure shows the positions at which the protons cross the galactic plane. The position of the galactic center is indicated with a cross and that of the solar system - with $\odot$.

The particle density in the vicinity of the 'source' looks uniform. At certain distance from the source, however, the protons tend to be contained in the high

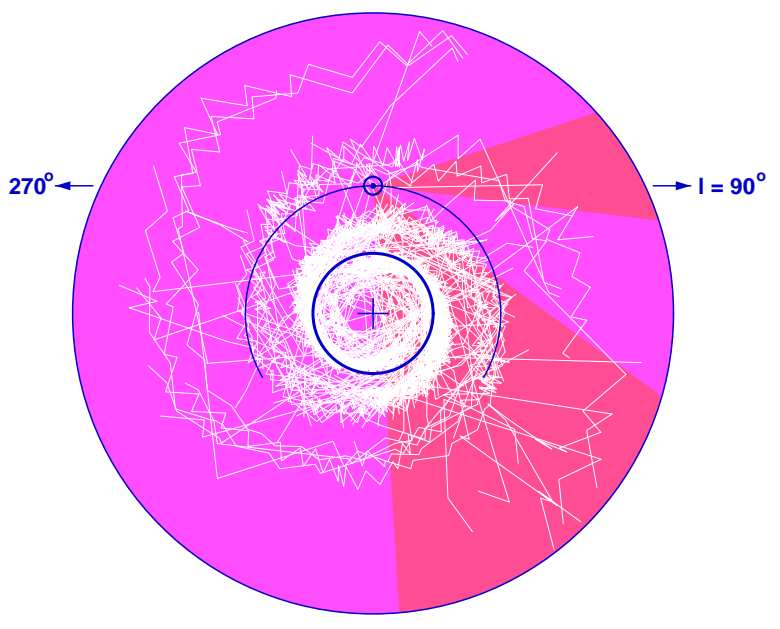

Figure 5: Positions at which $10^{18} \mathrm{eV}$ protons emitted isotropically at the $4 \mathrm{kpc}$ circle cross the galactic plane. The two red zones are the directions in which AGASA has observed increased flux of protons of similar energy. field regions and follow the large scale structure of the field. Occasionally they suffer a large angle deflection in the random field and migrate to a different magnetic 'arm'.

The two red zones are the directions in which the AGASA experiment has observed an excess of $10^{18} \mathrm{eV}$ cosmic rays: close to the galactic center (which is not visible from the location of AGASA) and in the direction of Cygnus 这列. The flux of cosmic rays from the direction of the galactic anticenter is smaller than the average. Figure 5 is not a model for the explanation of this observation - it is just an example of how organized magnetic fields modify the cosmic ray density.

The extreme example of particle deflection in the galactic field is due to Biermann [i] $\left[\begin{array}{l}1 \\ 1\end{array}\right]$. 
Using the astronomical observation that in all spiral galaxies the magnetic field has the same structure as in ours, Biermann uses the Parker spiral as a model for the halo field. Antiprotons injected from the solar system in any direction move always in the direction of the galactic North. If the extension of the halo field is of the order of one Mpc, even $10^{20} \mathrm{eV}$ antiprotons would eventually have directions close to the galactic North Pole and respectively to the Virgo cluster, which contains the most powerful active galaxy in the nearby Universe - M87. This is also an excellent example of how UHE cosmic rays could stream in a particular direction under the influence of magnetic fields that are coherent on Mpc scales.

\section{Discussion}

Many of the most recent analyses of the arrival directions of UHE cosmic rays account in one way or another for the particle deflections in magnetic fields. The results are, as expected in view of the numerous possible models and the limited observational statistics, often in contradiction to each other.

Tinyakov \& Tkachev, for example, find a correlation between the AGASA events above $4 \times 10^{19} \mathrm{eV}$ and a subset of $22 \mathrm{BL}$ Lac objects. The probability that the correlation is due to an isotropic arrival direction distribution is estimated to $2 \times 10^{-5}[\overline{2} \overline{0} \overline{0}]$. Furthermore, after the directions of the AGASA events are modified by propagation in a galactic magnetic field model the correlation seems to improve [2] $\left.\overline{1}_{1}^{1}\right]$. Since all known BL Lac objects are at distances exceeding $100 \mathrm{Mpc}$, the only possible conclusion is the the extragalactic magnetic field is very weak $-10^{-11} \mathrm{G}$ or less. The improvement of the correlation suggests the UHECR are indeed protons.

The UHECR correlation with BL Lac objects is widely disputed on different grounds [iَ2 $2 \overline{2}]$, but it is one of the examples of a contemporary analysis that attempts to study not only the nature of UHECR, but also important conditions of the extragalactic space.

One of the most prominent features of the AGASA UHECR data is the very strong auto-correlation shown in terms of statistical significance in Fig. 6. When the angular distance between all possible pairs of events is examined there is a very strong peak at about $2^{\circ}$, which is comparable to the angular resolution of the detector. This distribution is discussed in many recent papers that relate it to the conditions of extragalactic space and some of the characteristics of the UHECR sources.

Sigl, Miniati \& Ensslin [203] create a model of the local Universe within $50 h^{-1} \mathrm{Mpc}$. The model uses the observed mass distribution and assigns magnetic field values and UHECR source densities that are proportional to the mass. The magnetic field reaches $10^{-7} \mathrm{G}$ in the dense regions, which could be associated with clusters of galaxies. Ultra high energy cosmic rays are then injected at the sources and propagated with account for the energy loss and the scattering in the magnetic field. Observers are located in regions with high $\left(10^{-7} \mathrm{G}\right)$ and low $\left(10^{-11} \mathrm{G}\right)$ fields. The overall isotropy of the 'detected' UHECR and the autocorrelation between simulated events are compared to the AGASA data and conclusions are drawn about the cosmic ray source density and the most likely magnetic field configuration. Magnetic fields of $10^{-7} \mathrm{G}$ in the vicinity of the cosmic ray sources and 
a source density of $10^{-4}-10^{-5} \mathrm{Mpc}^{-3}$ are the conditions that describe data best. This corresponds to a source luminosity of $10^{38} \mathrm{erg} \mathrm{Mpc}^{-3} \mathrm{~s}^{-1}$ with a large uncertainty. In the absence of magnetic field Blasi \& De Marco [24] find a source density of $10^{-5} \mathrm{Mpc}^{-3}$ and

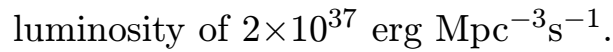

A series of papers by Yoshiguchi et

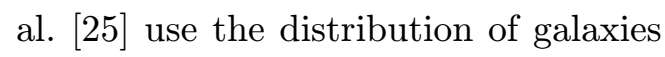
within the GZK sphere as a UHECR source distribution and propagate protons to the solar system including also the galactic magnetic field. The energy spectrum of the protons at arrival and their arrival directions are studied. The conclusion is that the arrival direction distribution (clustering) is not consistent with the assumption of strong magnetic fields $(>10 \mathrm{nG})$ in the GZK sphere. One the other hand, only fields of this magnitude can generate the observed large scale isotropy if the UHECR sources are associated with the most luminous galaxies. The source density which is consistent the most with obser-

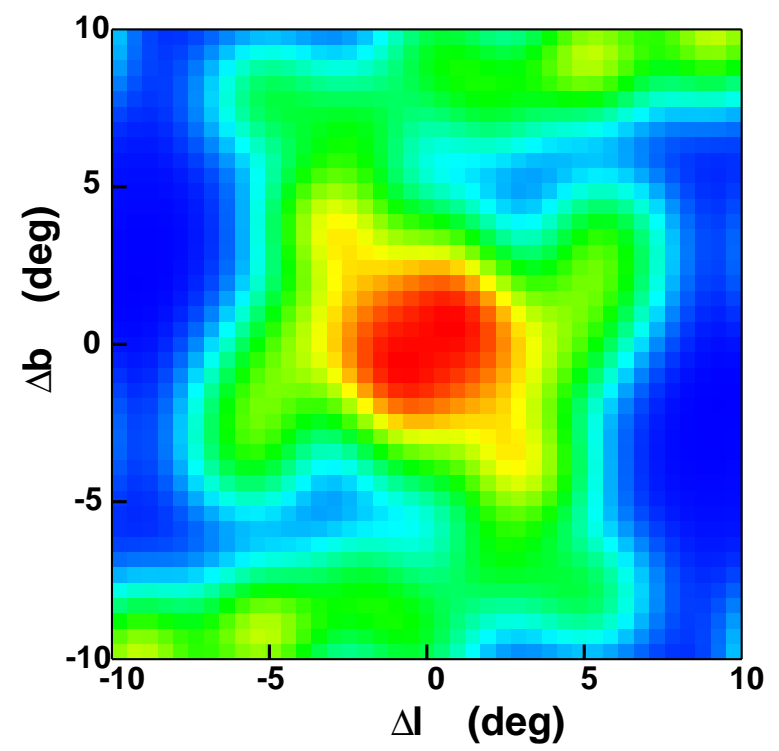

Figure 7: 2D autocorrelation in galactic latitude and longitude of the AGASA events above $4 \times 10^{19} \mathrm{eV}$. vations is $10^{-6} \mathrm{Mpc}^{-3}$, i.e. when only a fraction of the most luminous galaxies are sources of ultra-high energy cosmic rays. Each individual source has then to be more powerful by about an order of magnitude.

All papers that describe proton propagation in magnetic fields emphasize the fact that source positions are so smeared that one needs observational statistics much higher than the existing one to study the conditions of extragalactic space. The statistics expected from the Auger observatory would be sufficient to answer most of the current questions.

An interesting analysis and interpretation of the AGASA results was presented by Teshima at the International Cosmic Ray Conference in Hamburg (2001). Instead of the usual autocorrelation function a two dimensional correlation function is extracted from all events above $10^{19} \mathrm{eV}$ that come from an area around the galactic anti-center. The result for energies above $4 \times 10^{19}$ is shown in Fig. 7. For most energy thresholds (all above $10^{19}$ $\mathrm{eV}$ ) the shape of the $2 \mathrm{D}$ correlation is quite similar. The suggestion is that the shape of the correlation is related to the direction of the galactic magnetic field, which bends the trajectories of the high energy protons in a specific way. A simulation of the propagation of protons in the Galaxy and studies of the shape of the $2 \mathrm{D}$ correlation as a function of the number of sources, their positions, and the field model [i2 $\overline{6} \overline{6}]$ did not prove this suggestion right or wrong. The statistics of UHECR is still too small to study complicated effects like this.

The hope is that in the near future we will have data from the new air shower exper-

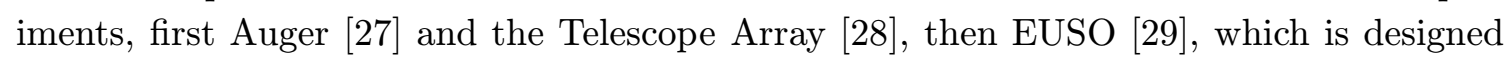




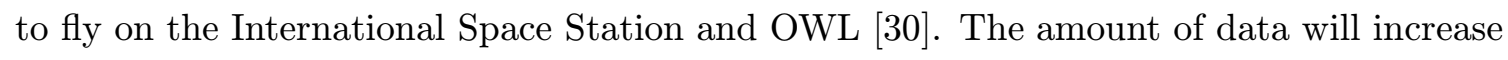
by orders of magnitude and the solution of the UHECR puzzle will also bring important information about the conditions in the nearby Universe.

\section{Acknowledgments}

The work of which this talk is based is performed in collaboration with J. Alvarez-Mũniz, R. Engel, D. Seckel and others. My research is funded in part by NASA grant NAG5-10919.

\section{References}

[1] G. Cocconi, Nuovo Cimento, 3, 1422 (1956)

[2] see P. Bhattacharjee \& G. Sigl, Phys. Rep., 327, 109 (2000) for a review

[3] see, e.g. T. Weiler, Astropart. Phys., 11, 303 (1999)

[4] K. Greisen, Phys. Rev. Lett., 16, 748 (1966); G.T. Zatsepin \& V.A. Kuzmin, LETP lett. 4, $78(1966)$

[5] J. Linsley, Phys. Rev. Lett., 10, 146 (1963)

[6] M. Takeda et al., Phys. Rev. Lett., 81, 1163 (1998)

[7] Y. Uchihori et al., Astropart. Phys., 13, 151 (2000)

[8] HiRes Collaboration, T. Abu-Zayyad et al., astro-ph/0208243; astro-ph/0208301

[9] D.J. Bird et al., Phys. Rev. Lett., 71, 3401 (1993)

[10] M. Nagano \& A.A. Watson, Rev. Mod. Phys., 72, 689 (2000)

[11] R.J. Protheroe \& P.L. Biermann, Astropart. Phys., 6, 45 (1996)

[12] R.J. Protheroe and T. Stanev, Phys. Rev. Lett., 77, 3708 (1996)

[13] V.S. Berezinsky \& S.I. Grigorieva, A\&A, 199, 1 (1988)

[14] V.S. Berezinsky, A.Z. Gazizov \& S.I. Grigorieva, astro-ph/0204357

[15] P.P. Kronberg, Rep. Progr. Phys., 58, 325 (1994); T.E. Clarke, P.P. Kronberg \& H. Böringer, Ap. J. Lett., 547, L111 (2001)

[16] T. Stanev et al., Phys. Rev. D62:093005 (2000)

[17] T. Stanev, D. Seckel \& R. Engel, Phys. Rev. D68:103004 (2003)

[18] N. Hayashida et al., Astropart. Phys., 10, 303 (1999)

[19] P.L. Biermann et al., Nucl. Phys. B (Proc. Suppl.) 87, 417 (2000)

[20] P.G. Tinyakov \& I.I. Tkachev, JETP Lett., 74, 445 (2001)

[21] P.G. Tinyakov \& I.I. Tkachev, Astropart. Phys., 18, 165 (2002)

[22] see, e.g. N. Wyn Evans, F. Ferrer \& S. Sarkar, Phys. Rev. D67:103005 (2003)

[23] G. Sigl, F. Miniati \& T.A. Ensslin, Phys. Rev. D68: 043002 (2003)

[24] P. Blasi \& D. De Marco, astro-ph/0307067 
[25] H. Yoshiguch et al., Ap.J., 586, 1211 (2003); H. Yoshiguch, S. Nagataki \& K. Sato, Ap.J., 592, 311 (2003); 596, 1044 (2003)

[26] J. Alvarez-Muñiz, R. Engel \& T. Stanev, Ap.J., 572, 185 (2002)

[27] http://www.auger.org

[28] http://www-ta. icrr.u-tokyo.ac.jp

[29] http://www.euso-mission.org

[30] http://owl.gsfc.nasa.gov 